\title{
Cutaneous Ulcers, Delusional Disorders and Munchausen by Proxy: From Somatic to Psychological Diagnosis and Approach
}

Marcello Menta Simonsen NICO ${ }^{1}$ and Alexandre Jack DWAN ${ }^{2}$

${ }^{1}$ Department of Dermatology and ${ }^{2}$ Department of Psychiatry, University of São Paulo, Rua Itapeva 500-3a, 01332-000 São Paulo, SP, Brazil. E-mail: mentanico@hotmail.com

Accepted Feb 11, 2022; Epub ahead of print Feb 11, 2022

Acta Derm Venereol 2022; 102: adv00654. DOI: 10.2340/actadv.v102.677

Dermatological diagnoses of self-inflicted mucocutaneous lesions should be followed by determination of the underlying psychopathology, so that the most appropriate and effective treatment can be offered. A purely dermatological approach is of no help in most instances. Artificial cutaneous lesions can be produced through various psychopathological mechanisms, including anxiety, depression, obsessive-compulsive, delusional, and factitious dermatological disorders (formerly called dermatitis artefacta or factitious dermatitis) $(1,2)$. Treatments based on the underlying psychopathology with possible subsequent prescription of a psychotropic drug, instead of relying on a purely dermatological diagnosis (factitious lesion, neurotic excoriation, lichen simplex chronicus, etc.), can lead to an improved outcome (1).

We report here a case of obviously artificially produced skin lesions, which we believe were maintained through a psychopathological mechanism that is not frequently observed in otherwise healthy adult patients.

\section{CASE REPORT}

A previously healthy, 45-year-old, married Brazilian man presented with incapacitating symptoms of stinging, burning, and pain on the skin on his back. He related the symptoms to the presence of "worms" infecting his skin. Clinical examination revealed several bizarre-shaped ulcers and scars, which were clearly caused by manipulation of the skin.

The patient's 40-year-old wife actively participated in the medical interview, and vividly corroborated her husband's reports, including the presence of "worms". She brought bandages, gauze packs, and other dressing material to the appointment, carefully removed the dressings that covered his wounds, and applied ointments and new adhesive tape to each lesion after examination (Fig. 1). Both the patient and his wife acknowledged that all the lesions were produced by her poking and squeezing the skin as she tried to "find and "extract the worms", "always following his instructions".

Folie a deux delusional infestation was the initial diagnosis proposed by the dermatology team, and patient was started on risperidone $1 \mathrm{mg}$. The dosage was increased gradually up to 3 $\mathrm{mg}$, but there was no improvement in symptoms. Olanzapine was subsequently tried, also with no favourable results. Amitriptyline was also used, as well as oral pain medications and several topical treatments. The patient and his wife continued to attend consultations, always maintaining the same reports and behaviour.

At a further visit, the psychiatrist in our group interviewed the couple. Psychiatric evaluation revealed that the patient had a tolerant attitude towards the alleged symptoms: despite verbal report of intense distress, he showed no physical signs of restlessness, and maintained a calm demeanour, kept his voice tone low and re-

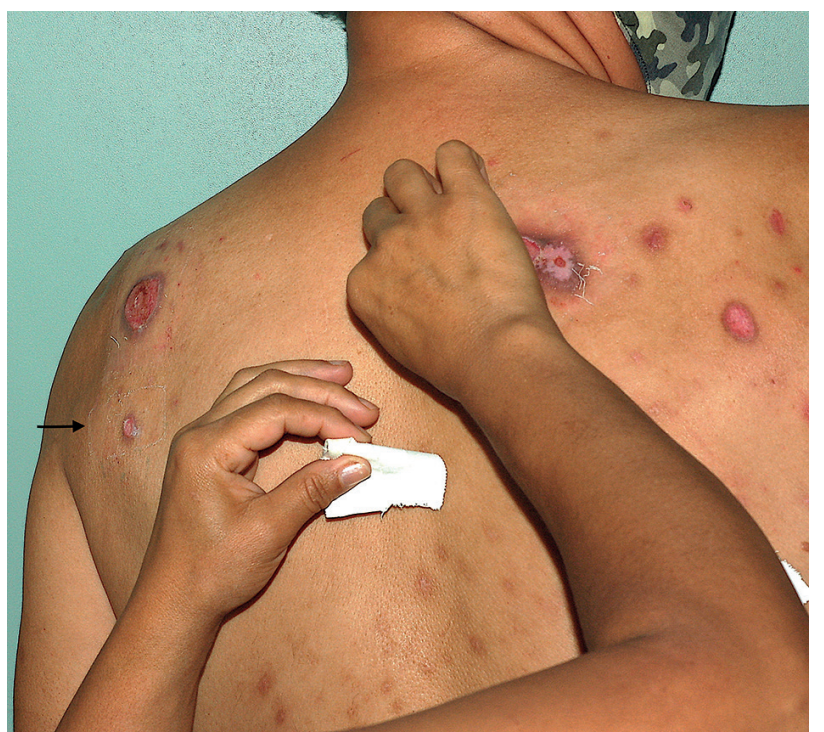

Fig. 1. Patient's wife removing the dressing of one of the patient's skin ulcers at the start of the consultation. The dressings of the other lesions have just been removed (arrow).

mained unfazed throughout medical evaluations, although always complaining of the symptoms. No sign of delusional, depressive, manic, mixed-state episode, or external gain were detected.

The patient's emotional detachment was even more apparent when contrasted with his wife's behaviour, which was largely the same as during dermatological evaluation. She was considerably more engaged, insisted on the presence of parasites, attempted to describe them in detail, made remarks about her husband's suffering, and left little room for other topics to be brought to attention.

Despite the failure of multiple treatment modalities, the patient and his wife have continued to attend follow-up visits and a good patient-doctor relationship is progressively being established with the psychodermatology team.

\section{DISCUSSION}

The differential diagnoses in psychodermatological cases involving more than 1 person include delusional folie a $\operatorname{deux}$ (in which a dominant delusional patient "convinces" the other person about an infestation) (3), and Munchausen syndrome by proxy (a psychological disorder marked by attention-seeking behaviour by a caregiver through those who are in their care) (4).

The clinical lesions in the current patient were not of particular interest, they were obviously factitious; what made this case peculiar was the dynamics of the consultation. The fact that the wife openly acknowledged pro- 
ducing the lesions, patiently took care of her husband's wounds, and supported his claims, initially made us think of a type of folie a deux that had began with a delusional state in the husband (1). At further visits, however, their behaviour and speech led us to a different hypothesis.

On closer evaluation, the phenomenology of symptoms did not support the hypothesis of delusional disorder. Although the presence of parasites was initially mentioned repeatedly, the patient had no issue in accepting the possibility of a psychiatric diagnosis for his condition, which showed remarkable mental awareness, in contrast to the usual resistance seen in delusional cases. Actual perceptual disturbances were also not detected.

On the other hand, the patient's wife assimilated his complaints and proceeded to act accordingly, reinforcing the situation with his consent. In contrast to the initial hypothesis of delusional disorder and despite maintaining claims of parasitic infestation of her husband, she did not present with additional elements of psychotic symptoms, her affect remained adequate and coherent during all encounters, and she did not react with the usual hostility when other possible causes of the patient's complaints were suggested. In addition, she denied use of alcohol or any other recreational drugs, which could provide another explanation for their peculiar behaviour and perception. Since we saw no likely benefit of a pharmacological approach, she received no medication.

Over time, the significance of the symptoms increased, to become more important in her perspective than in the patient's own.

Symptoms not associated with identifiable physiological changes have long posed a challenge to the medical community. In recent times, the diagnosis of hysteria has evolved to a multitude of subcategories, and current psychiatric diagnostic classification has been met with challenging questions, particularly the Diagnostic and Statistical Manual $5^{\text {th }}$ edition (DSM-5) from the American Psychiatric Association $(5,6)$. Criticism ranges from excessive psychological emphasis on diagnostic criteria to presuming causality without adequate supporting evidence.

In the current case, we aimed first to either confirm or rule out delusional disorders, which would involve a clear treatment path with antipsychotics. Following this initial step and taking into account additional informa- tion gathered, our diagnostic hypothesis moved on to the possibility of Munchausen by proxy (or factitious disorder imposed on another, according to the DSM-5) and the approach to the case was adjusted accordingly, namely to minimize iatrogenics and focus on behaviour modification (7). Factitious disorder imposed on another is more commonly seen in children or in debilitated elderly people, which makes the current case even more unusual (4).

This is an interesting example of a bona fide psychocutaneous disease that was initially very difficult to manage because the psychopathology involved did not fit into the usual categories more commonly seen by dermatologists. The presence of a psychiatrist in the consultation was pivotal to establishing an appropriate diagnosis, since dermatologists are not accustomed to such cases. A multidisciplinary approach was of great value, as it often is in psychodermatology.

At time of writing, the patient is being treated with topical antibiotic ointments as well as oral placebo pills, and he reports very slow, but continuous, improvement in the skin stinging. As stated, a good doctor-patient relationship is slowly being established, which may enable the couple to be referred to more specific therapies, such as psychotherapy, in the future.

\section{REFERENCES}

1. Wong JW, Koo JYM. Psychopharmacological therapies in dermatology. Dermatol Online J 2013; 19: 18169.

2. Carnahan KT, Jha A. Factitious disorder. 2021 Aug 9. In: StatPearls [Internet]. Treasure Island (FL): StatPearls Publishing; 2021 [accessed Oct 27, 2021] Available from https:// pubmed.ncbi.nlm.nih.gov/32491479.

3. Martins AC, Mendes CP, Nico MM. Delusional infestation: a case series from a university dermatology center in São Paulo, Brazil. Int J Dermatol 2016; 55: 864-868.

4. Glaser D. Fabricated or induced illness: From "Munchausen by proxy" to child and family-oriented action. Child Abuse Negl 2020; 108: 104649.

5. American Psychiatric Association. (2013). Diagnostic and statistical manual of mental disorders (5th edn). American Psychiatric Publishing, Washington DC and London, England.

6. Bransfield RC, Friedman KJ. Differentiating psychosomatic, somatopsychic, multisystem illnesses, and medical uncertainty. Healthcare (Basel) 2019; 7: 114.

7. Weber B, Gokarakonda SB, Doyle MQ. Munchausen Syndrome. 2021; 31. In: StatPearls [Internet]. Treasure Island (FL): StatPearls Publishing; 2021. [accessed Oct 27, 2021] Available from https://pubmed.ncbi.nlm.nih.gov/30085541. 